\title{
Dipole radiation model for surface roughness scattering in hollow-core fibers
}

\author{
Eric Numkam, Francesco Poletti and David J. Richardson \\ Optoelectronics Research Centre, University of Southampton, Highfield Campus, SO17 1BJ, Southampton, UK \\ ernf1g10@orc.soton.ac.uk
}

\begin{abstract}
We propose a physical model based on dipole radiation to estimate surface roughness scattering loss in microstructured and hollow-core photonic bandgap fibers. Angular distribution of scattered power and overall loss values can be calculated.

OCIS codes: (060.4005) Microstructured fibers; (240.5770) Roughness; (290.5880) Scattering, rough surfaces
\end{abstract}

\section{Introduction}

Hollow-core photonic bandgap fibers (HC-PBGFs) which confine light in an air-core and guide it by means of a photonic bandgap have attracted tremendous scientific interest over the past two decades. One of their most attractive features is the potential to achieve propagation losses below those of conventional single mode fibers, which would make HC-PBGFs very attractive for next-generation telecoms networks.

One distinct feature of these fibers is the large number of glass-air interfaces present within the structure. As Rayleigh scattering and bulk absorption play a negligible role in their overall loss, it has been found that roughness scattering from these interfaces imposes a fundamental limit to their attenuation [1]. Therefore, an accurate model of light scattering from rough air-glass surfaces can prove an essential tool in the design of ultralow-loss HC-PBGFs. A complex model based on coupled-mode theory and Green tensors was previously applied to solid core photonic crystal fibers (PCFs) but requires circular air holes and therefore cannot be applied to HC-PBGFs [2].

In this paper, we follow an approach pioneered by Rawson in standard step-index fibers [3], and formulate a light scattering model based on induced dipole radiation to analyze and predict scattering losses in fibers with air holes of any potential shape. The model gives insight on how the roughness power spectral density (PSD) affects the angular distribution of scattered power, allows quantitative predictions of surface scattering loss and how this depends on wavelength and various fiber design parameters.

\section{Model Formulation}

To introduce the model, we consider a waveguide consisting of a hollow glass tube of radius $\bar{r}$ and thickness $t$. Such a waveguide is an idealization of the core of a HC-PBGF, where thermodynamically excited surface capillary waves frozen-in during fiber draw pose a fundamental limit to the minimum surface roughness, with rms of the order of $\sim 0.1 \mathrm{~nm}$ [1]. Due to such a roughness the inner and outer wall boundaries depart from the ideal case and can be described in general terms as $r_{1}=\bar{r}+f_{1}\left(s_{1}, z\right)$, and $r_{2}=\bar{r}+t+f_{2}\left(s_{2}, z\right)$ respectively, where $f_{i}$ are small scale perturbations and $s_{i}$ the arc length along the interface.

In this model we consider any roughness "bump" on the waveguide walls (either positive i.e. made of glass or negative i.e. made of air) as an induced electric dipole of volume $d V=\left|f_{i}\right| d s_{i} d z$ and polarizability $\alpha$. In general $\alpha$ will depend on the shape and 'sign' of the bump, and in high index-contrast surfaces it is different for orthogonal and parallel field polarizations. However, Johnson et al. have computed the polarizability for different shapes and index contrasts [4], and found that for silica-air at infrared wavelengths (contrast between $\sim 0.5$ and 2), it is acceptable to neglect the effect of field discontinuities and only consider the sign variation, that is $\alpha=\alpha_{0} \operatorname{sgn}\left(f_{i}\right)$. Every dipole on the surface will have an induced dipole moment $\vec{p}=\alpha \mathrm{dV} \overrightarrow{\mathrm{E}_{0}}$ where $\overrightarrow{\mathrm{E}_{0}}$ is the incident field at the dipole position. We consider the field scattered out of a section of length $2 \mathrm{~L}$ of the waveguide at a point $\mathrm{P}$ on a distant sphere and in a plane making angle $\vartheta$ with the yz plane (see Fig.1(a)). We assume this section to be sufficiently long to contain all the major spatial spectral features of the roughness, but short enough so that guidance loss can be neglected throughout the section. The field scattered at $\mathrm{P}$ by a single dipole is given by:

$$
\mathrm{d} \overrightarrow{\mathrm{E}_{\mathrm{s}}}=\frac{1}{4 \pi \varepsilon_{0}} \frac{k_{0}^{2}}{R}(\vec{r} \times \vec{p}) \times \vec{r}
$$

where $R$ is the distance to the probing point $\mathrm{P}$ and $k_{0}$ is the vacuum wavenumber. Expanding the incident field in terms of its components perpendicular $\left(E_{0 \perp}\right)$ and parallel $\left(E_{0 \|}\right)$ to the Pz plane, we obtain:

$$
d \overrightarrow{E_{s, l}}=\frac{k_{0}^{2}}{4 \pi \varepsilon_{0} R} \alpha_{0} f_{i}(s, z) d s d z\left[E_{0 \perp} \vec{v}+\left(E_{0 z} \sin \phi-E_{0 \|} \cos \phi\right) \vec{u}\right]
$$


(a)

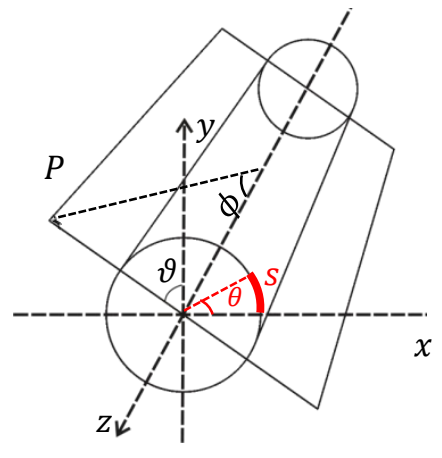

(b)

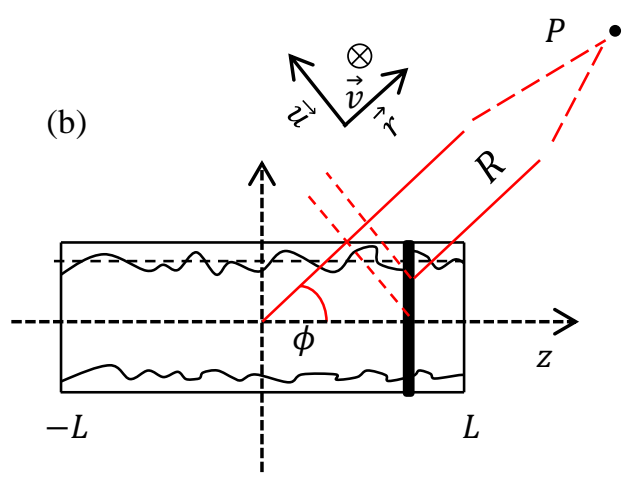

Fig.1: (a) Illustration of observing point $\boldsymbol{P}$ with respect to the fiber axes; (b) relative phase between fields scattered from different positions along the waveguide.

The field scattered by different points on the glass-air surface reaches $\mathrm{P}$ with different phases. Taking the field originating from the point $\left(\mathrm{z}=0, \vartheta+\frac{\pi}{2}\right)$ as a reference (Fig. 1(b)), the phase difference of all other rays is given by $\varphi(\phi, \vartheta)=\beta z-k_{0} z \cos \phi-k_{0} \bar{r} \cos (\theta-\vartheta) \sin \phi$, where $\beta$ is the mode propagation constant and $\theta$ the azimuthal position of the scattering point $\left(s_{i} / \bar{r}\right)$.

The total field scattered at $\mathrm{P}$ is therefore:

$$
\overrightarrow{E_{s}}=\sum_{i} \int_{z=-L}^{L} \oint d \overrightarrow{E_{s, l}} \exp (-i \varphi)
$$

Assuming that the azimuthal component of the surface roughness does not considerably contribute to scattering losses [1], the above integration along $\mathrm{z}$ yields the Fourier transform $\tilde{F}$ of the roughness, and the result is:

$$
\begin{array}{r}
\overrightarrow{E_{S}}(\phi, \theta)=\sum_{i} \frac{k_{0}^{2}}{4 \pi \varepsilon_{0} R} \tilde{F}\left(\beta-k_{0} \cos \phi\right)\left[\vec{v} \oint E_{0 \perp} e^{i k_{0} \bar{r} \cos (\theta-\vartheta) \sin \phi} d s_{i}\right. \\
\left.+\vec{u} \oint\left(E_{0 z} \sin \phi-E_{0 \|} \cos \phi\right) e^{i k_{0} \bar{r} \cos (\theta-\vartheta) \sin \phi} d s_{i}\right]
\end{array}
$$

This expression shows that each spatial frequency of the roughness governs the scattering intensity at a specific direction $\phi$. The angularly resolved scattered power per steradian in the $\phi$ direction can be obtained from equation (4) and integration along $\vartheta$ and $\phi$ yields the scattering loss coefficient: $\alpha_{l}=\frac{1}{2 L} \int_{\vartheta=0}^{2 \pi} \int_{\phi=0}^{\pi} \frac{1}{2} \operatorname{cn} \varepsilon_{0}\left|E_{s}\right|^{2} R^{2} \sin \phi d \vartheta d \phi$.

\section{Results and discussion}

The model presented above was initially applied to calculate the scattering from both inner and outer surfaces of a simple hollow tube with a radius $\bar{r}$ of $10 \mu \mathrm{m}$, roughly corresponding to the core of a 19-cell HC-PBGF, and a thickness $t$ chosen to satisfy the antiresonant condition: $t=\lambda / 4 \sqrt{n_{\text {Glass }}^{2}-n_{\text {Air }}^{2}}$ [3]. Using a semi-analytical transfer matrix method [5] we calculated all the field components of the x-polarized fundamental $H E_{11}$ mode. We then employed the well-known expression for the roughness PSD of frozen-in surface capillary waves, $S=k_{B} T_{g} / 4 \pi \gamma \kappa$ [1], to compute the scattered power per unit steradian on a distant sphere enclosing the scattering fiber section. To avoid the singularity in the roughness spectrum at low spatial frequencies we introduce a low frequency cut-off at $\sim 0.1 \mu^{-1}$. Spatial frequencies lower than this value are then assumed to have the same power spectral density.

The calculated distribution of scattered power on a distant sphere and the average scattered power as a function of escaping angle $\phi$ along the propagation direction (or effective index) are shown in Fig. 2(a) and (b), respectively. Due to the nature of the roughness spectrum, the electric field is preferentially scattered in the forward direction. The peaks and dips in the figures result from constructive and destructive interference at the probing point, a feature which is also confirmed by coupled mode theory based models on all-solid fibers [6]. Obviously, simulations show different scattering patterns for different modes of the waveguide. Of particular interest is the loss variation with changes in waveguide parameters and wavelength. It has previously been argued through dimensional analysis that roughness scattering loss in HC-PBGFs must be proportional to $\lambda^{-3}$ [1]. Our model confirms this trend but it indicates that the $\lambda^{-3}$ wavelength dependence results from the enlarged core size required to operate at longer wavelengths, rather than from the roughness contribution. Fig.2(c) illustrates this point by showing how the loss 
changes with wavelength when (i) the core radius $\bar{r}$ is kept fixed but $t$ is changed so that antiresonance is achieved at the chosen wavelength, and (ii) both $\bar{r}$ and $t$ are scaled proportionally to the wavelength.

Another interesting result of our study is that the optimum tube thickness minimizing the leakage loss is different from that minimizing the scattering loss. For example, in a tube with antiresonant thickness $t$ and rms roughness of $0.1 \mathrm{~nm}$ our model predicts a scattering loss of $0.13 \mathrm{~dB} / \mathrm{km}$ at $1.55 \mu \mathrm{m}$. This value is nearly halved when the optimum thickness of $1.27 t$ is chosen, clearly indicating that a good model for studying roughness scattering can be extremely helpful in designing lower loss air core fibers such as HC-PBGFs.

(a)

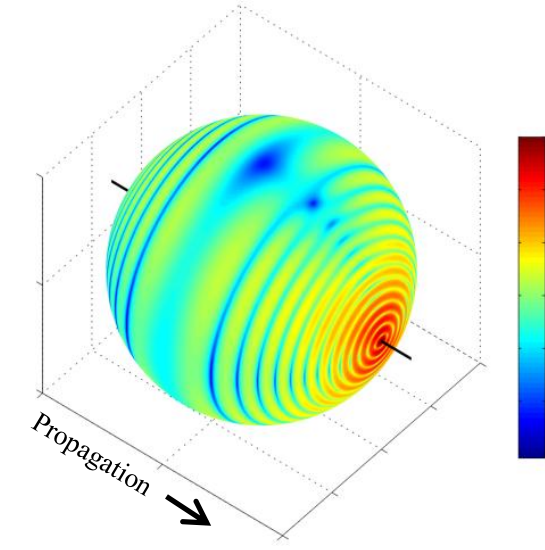

(c)

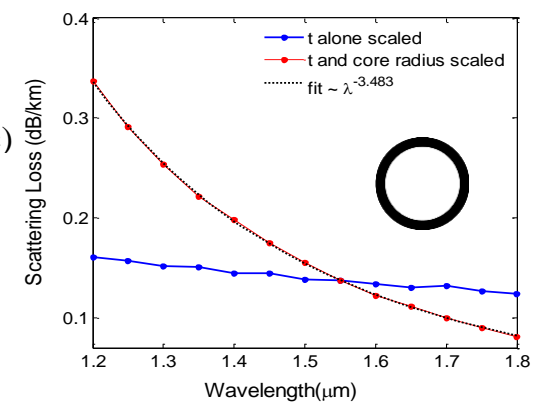

(b)

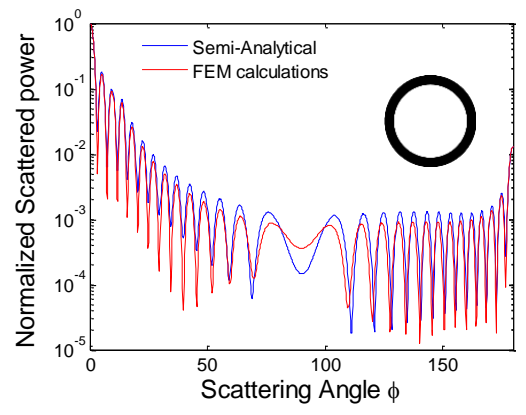

(d)

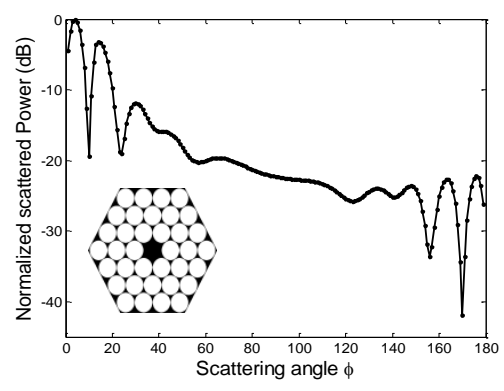

Fig.2: (a) Computed distribution of scattered field from roughness on both surfaces of a tube waveguide on a distant sphere; (b) Average angular distribution of scattered power in a tube waveguide from semi-analytical and finite element calculations; (c) wavelength dependence of total scattering loss, and (d) angular distribution of scattered power in the solid-core PCF of [2] (2.85 $\boldsymbol{\mu m}$ pitch and 2.74 $\boldsymbol{\mu m}$ hole diameter).

For more complex waveguides than a simple hollow tube, numerical methods need to be employed to obtain the electric field components at the glass-air interfaces required in equation (4). As a first step towards the study of HCPBGFs, we have developed a finite element method (FEM) based approach to study solid-core PCFs with a multitude of circular air-holes. Accuracy of our chosen numerical approach was first validated on the previous hollow tube fiber (Fig. 2(b)), and then applied to the small core PCF experimentally studied by Roberts et al. [2]. Although our model currently assumes an air rather than glass cladding outside the microstructure, the results are in very good qualitative agreement (Fig. 2(d)). Comparison with the single tube result in Fig. 2(b) shows that the presence of multiple holes considerably smoothes out the strong interference pattern.

\section{Conclusion}

In conclusion we have proposed a model of light scattering from surface roughness in a variety of holey waveguides. The scattering field distribution was computed analytically for a simple tube waveguide and numerically with FEM solvers for more complex structures. The impact of the full roughness spectrum on the overall scattering loss was assessed and the wavelength dependence of scattering loss studied. We believe that this model will ultimately allow deeper understanding of the scattering process in HC-PBGFs, allowing the potential design of ultralow-loss HCPBGFs for telecoms applications. This work was supported by the EU program FP7-ICT MODE-GAP.

\section{References}

[1] P. J. Roberts et al., "Ultimate low loss of hollow-core photonic crystal fibers,"Opt. Exp., vol. 13, pp. 236-244, 2005

[2] P. J. Roberts et al, "Loss in solid-core photonic crystal fibers due to interface roughness scattering," Opt. Exp., vol. 13, pp. 7779-7793, 2005

[3] E. G. Rawson "Analysis of scattering from fiber waveguides with irregular core surfaces", Applied Optics 13. No. 10, pp2370 - 2377, (1974)

[4] S.G. Johnson et al, "Roughness losses and volume-current methods in photonic-crystal waveguides", Appl. Phys. B 81, 283-293 (2005)

[5] Pochi Yeh, Amnon Yariv and Emmanuel Marom, "Theory of Bragg Fiber", J.Opt.Soc.Am 68, No 9, 1196-1201 (1978)

[6] D. Marcuse, Theory of Dielectric Optical waveguides, 2nd ed. San Diego, CA; Academic Press 1991, chap 4. 